\title{
BMJ Open Association of smoking reduction and mortality: protocol for a systematic review and meta-analysis of longitudinal observational studies
}

\author{
Henri-Jean Aubin (D) , ${ }^{1}$ Laetitia Ali Oicheih, ${ }^{2}$ Sonia Gabriel, ${ }^{2}$ Ivan Berlin (1) ${ }^{3}$
}

To cite: Aubin H-J, Ali Oicheih L, Gabriel S, et al. Association of smoking reduction and mortality: protocol for a systematic review and meta-analysis of longitudinal observational studies. BMJ Open 2021;11:e039483. doi:10.1136/ bmjopen-2020-039483

- Prepublication history and additional material for this paper are available online. To view these files, please visit the journal online (http://dx.doi. org/10.1136/bmjopen-2020039483).

Received 16 April 2020 Revised 03 December 2020 Accepted 02 January 2021

Check for updates

(c) Author(s) (or their employer(s)) 2021. Re-use permitted under CC BY-NC. No commercial re-use. See rights and permissions. Published by BMJ.

${ }^{1}$ Département de psychiatrie et d'addictologie, Hopital Paul Brousse, Villejuif, Île-de-France, France

${ }^{2}$ CESP, INSERM, Université ParisSaclay, Villejuif, Île-de-France, France

${ }^{3}$ Pharmacology, Hopital PitieSalpetriere, Paris, Île-de-France, France

Correspondence to Dr Henri-Jean Aubin; henri-jean.aubin@aphp.fr

\section{ABSTRACT}

Introduction Strong evidence shows that smoking cessation decreases mortality. Much less is known regarding the association between reduction in cigarettes per day (CPD) and mortality. The primary aim of this systematic review is to compare the mortality risk between smokers achieving a sustained reduction of CPD and smokers maintaining their smoking rate. The secondary aims are to compare the mortality risk between smokers achieving complete, sustained smoking cessation and (1) smokers maintaining their smoking rate and (2) smokers who achieved a sustained reduction in smoking rate. Methods and analysis MEDLINE, Web of Sciences and Embase will be searched using a prespecified search strategy, up to 23 November 2020, and will be limited to studies published in English and in French. Longitudinal observational studies using individual data including smokers with at least two distant CPD assessments and a follow-up period of systematic mortality data recording will be included. The main outcome will be the allcause mortality. The secondary outcome will be specific mortality. The Newcastle-Ottawa Scale will be used to assess the risk of bias of individual studies. Outcomes will be analysed using HRs. All other outcomes' effect size reported in included studies will be converted in HRs using validated methods.

Ethics and dissemination We intend to publish the results of our review in a peer-reviewed journal and to present the findings at national and international meetings and conferences.

PROSPERO registration number CRD42019138354.

\section{INTRODUCTION}

\section{Rationale}

Increased mortality among cigarette smokers has long been well established, reaching two to three times the mortality rate of never smokers. Smoking results in the reduction in life span by an average of 10 years. ${ }^{1-6}$ The main causes of excess mortality among smokers are neoplastic, vascular, respiratory and other smoking-induced diseases. ${ }^{347}$ Allcause mortality provides a measure of the excess mortality attributable to smoking that integrates all these causes and also captures

\section{Strengths and limitations of this study}

- The systematic review will be conducted and reported according to the Preferred Reporting Items for Systematic Reviews and Meta-Analyses guidelines.

- Exposure will be defined using at least two distant cigarettes per day assessments.

- The meta-analysis associated with this review will allow quantifying the magnitude of the association between smoking reduction and mortality compared with maintained smoking intensity and smoking cessation.

- Heterogeneity, particularly differences in exposure and follow-up durations, may limit the strengths of the associations and conclusions.

- As evidence will be derived from observational studies, a conclusive answer on causality will not be provided.

mortality related to currently unidentified associations of smoking with health issues. ${ }^{3}$ A clear dose-response relationship between smoking intensity (ie, cigarettes per day (CPD)), duration of smoking and mortality has consistently been reported. ${ }^{25689}$

Smoking cessation reduces the risk of major chronic diseases and extends life expectancy by decreasing all-cause mortality. ${ }^{1-4} \quad 10 \quad 11$ Therefore, complete and definitive smoking cessation as soon as possible is considered the best avenue for smokers. ${ }^{361011}$ However, while well aware of this public health message, many smokers continue to smoke, probably because they feel unable or are unwilling to quit. ${ }^{12}$ For these smokers, harm reduction or harm minimisation strategies have been suggested, ${ }^{12}$ some of which being increasingly popular (ie, by using electronic nicotine delivery systems) ${ }^{13}{ }^{14}$ but for which the effectiveness in reducing the mortality risk compared with unchanged cigarette smoking rate remains to be comprehensively assessed.

According to the latest Surgeon General report, ${ }^{3}$ reductions in the number of 
cigarettes smoked per day are much less effective than smoking cessation in reducing the mortality associated with smoking. The reality or the extent of such a possible benefit of smoking reduction in avoiding smokingrelated mortality risks has however not been analysed in the report. A qualitative review reported in 2006 that a reduction in the number of CPD is associated with an increased probability of future smoking cessation but was unable to conclude about a change in disease risk. ${ }^{15} \mathrm{~A}$ systematic review published the following year examined the possible health benefit of reduced tobacco consumption and concluded that smoking reduction improves cardiovascular risk factors and respiratory symptoms and reduces the incidence of lung cancer. ${ }^{16}$ However, the review found no evidence of an association between a reduction in CPD (compared with unchanged CPD) and all-cause or most specific mortality rates except for a borderline significant decline in risk of tobacco-related cancers. It is noteworthy that the analysis of the association between reduction in CPD and mortality relied on a single observational study in this review. ${ }^{17}$ Since its publication in 2007, a number of additional studies reported contrasted findings regarding the extent of the possible association of reduction in CPD and mortality. ${ }^{18-22}$ The purpose of our review protocol is to update and synthesise the currently available data regarding the association between smoking reduction and mortality.

\section{Objectives}

The primary aim of this systematic review is to compare the mortality risk between smokers achieving a sustained reduction of CPD and smokers maintaining their smoking rate. The secondary aims are comparing mortality risk between smokers achieving complete, sustained smoking cessation, and (1) smokers maintaining their smoking rate $(\mathrm{CPD}),(2)$ and smokers achieving a sustained reduction. Depending on the CPD categories reported in the studies included in the review, comparison of the mortality risk between different levels of CPD reduction will be performed if appropriate.

\section{METHODS}

This protocol has been prepared according to the Preferred Reporting Items for Systematic Reviews and Meta-Analyses Protocols guidelines. ${ }^{23} 24$

\section{Eligibility criteria}

Studies (published in English or in French) will be selected according to the criteria outlined below.

\section{Study design}

We will include longitudinal observational studies using individual level data; reports using aggregated data will be excluded. Figure 1 is a schematic diagram of data on participants (smokers), exposure (CPD change categories: maintainers, reducers, quitters) and outcome

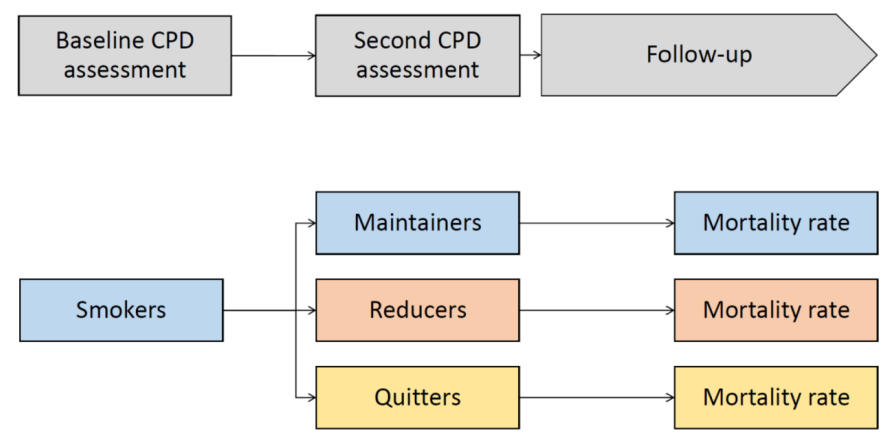

Figure 1 Schematic diagram of participants (smokers), exposure (CPD change categories: maintainers, reducers, quitters) and outcome (mortality rate). CPD, cigarettes per day.

(mortality rate) required for the eligible studies in the review.

\section{Participants}

Current smokers at baseline.

\section{Exposure}

The primary exposure of interest is cigarette smoking, expressed as the number of CPD, or as a CPD category (eg, 1-10, 11-20 and >20). CPD can be self-reported. CPD has to be measured at least twice: at baseline, and at a second and distant assessment. Individual change in CPD (or in CPD category) should have been assessed and reported.

\section{Comparators}

Three groups of participants' outcomes will be compared:

- Smokers having reduced their smoking rate (CPD) at a second assessment.

- Smokers having maintained (or increased) their smoking rate at a second assessment.

- Smokers having quit smoking at a second assessment.

\section{Outcome}

The main outcome will be all-cause mortality. Included studies must have systematically collected mortality records during a follow-up. The secondary outcome will be system specific mortality (ie, cardiovascular, cancer, respiratory and other...).

\section{Information sources}

We will search MEDLINE (1948 onwards) and EMBASE (1980 onwards). To ensure literature saturation, we will scan the reference lists of included studies or relevant reviews identified through the search. We will also search the authors' personal files to make sure that all relevant material has been captured.

\section{Search strategy}

The search will be performed up to 23 November 2020, and be limited to studies published in English or in French. The MEDLINE search strategy is included as follows: (smok*[Title] OR cigarette*[Title] OR tobacco[Title]) AND cigarette*[Abstract]) AND 
(mortality[Title/Abstract] OR death[Title/Abstract]) AND reduction*[Title/Abstract].

Web of Sciences and EMBASE searches will be started after completion of the MEDLINE search with subject headings and syntax of Web of Sciences and EMBASE. Further, the authors will check for additional relevant cited articles if not identified previously.

\section{Study records}

Data management

Literature search results will be uploaded and stored in Zotero (https://www.zotero.org); duplicates will be removed.

\section{Selection process}

At least two review authors will independently screen the titles and abstracts according to the eligibility criteria. Full reports with uncertain eligibility criteria will be checked. Identified full reports will be again checked for eligibility criteria before including them into the review. Online supplemental material 1 will be consulted if the information provided in the main published article is insufficient to assess whether or not the eligibility criteria are met. Between-review author disagreements will be resolved through discussion. Reasons for excluding trials will be recorded and reported.

\section{Data collection process}

Information will be extracted from all studies meeting eligibility criteria. Two reviewers will independently complete the data extraction form (see online supplemental table 1). Discrepancies will be resolved through discussion and/or consultation with the primary reviewer. We will contact study authors to resolve any uncertainties.

\section{Data items}

The data information form is structured in eight sections (online supplemental table 1):

- Study characteristics: setting, design, year of publication.

- Participants: characteristics of the study population such as country/region, age and sex distribution and the size of the population; the sampling methods used.

- Exposure/comparators: date of (and age at) baseline and second CPD assessment, CPD categories definition, CPD reducer definitions, health status at the beginning of the follow-up period.

- Outcomes timing/follow-up: date of (and age at) end of follow-up, duration of follow-up, specific mortality reported.

- Statistical analyses: the method for the main statistical analysis, the main effect measure, the magnitude of effect, with CIs will be recorded. We will record unadjusted and maximally adjusted estimates as appropriate and we will note the covariates used.

- Newcastle-Ottawa Scale (NOS) for cohort studiesscoring details in the three dimensions:

- Selection assessment.
- Comparability assessment.

- Ooutcome assessment.

\section{Outcomes and prioritisation}

The primary outcome of interest will be the all-cause mortality rate. The secondary outcomes will be specific mortality rate. Specific mortality will be classified according to the 2014 Surgeon General's Report ${ }^{3}$ :

- Cancer: lung cancer; other cancers (cancers of the lip, pharynx and oral cavity, oesophagus, stomach, pancreas, larynx, cervix uteri (women), kidney and renal pelvis, bladder, liver, colon and rectum; also acute myeloid leukaemia.

- Cardiovascular and metabolic: coronary heart disease; other heart disease (includes rheumatic heart disease, pulmonary heart disease and other forms of heart disease); cerebrovascular disease; other vascular disease (atherosclerosis, aortic aneurysm and other arterial diseases).

- Respiratory: pneumonia, influenza, tuberculosis; chronic obstructive pulmonary disease (includes emphysema, bronchitis and chronic airways obstruction).

We expect to prioritise exposure definition, that is, the definition of CPD reduction: the primary analysis will use the study authors' main definition of CPD reduction, or if not defined, change to any lower CPD group will be used. Alternative reduction definitions will be used in secondary analyses.

\section{Risk-of-bias assessment (in individual studies)}

The NOS for cohort studies will be used to assess the risk of bias of individual studies. ${ }^{25}$ Briefly, this scale contains eight items, categorised into three dimensions including selection, comparability and outcome. For each item, a series of response options is provided. A star system allows a semiquantitative assessment of study quality, such that the highest quality studies are awarded a maximum of one star for each item with the exception of the item related to comparability that allows the assignment of two stars. The NOS ranges between 0 and 9 stars. Risk-of-bias assessment will be conducted independently by two reviewers. Discrepancies will be resolved through discussion and/or consultation with the primary reviewer.

\section{Data synthesis}

Outcomes will be analysed using HR. All other outcome effect size measures will be converted into HRs, using validated methods. ${ }^{26-28}$ We will conduct meta-analyses (using inverse variance weighting) to calculate a pooled effect estimate.

Statistical heterogeneity will be tested using the $\mathrm{I}^{2}$ statistic (0\%-40\%: might not be important; $30 \%-60 \%$ : may represent moderate heterogeneity; 50\%-90\%: may represent substantial heterogeneity; $75 \%-100 \%$ : considerable heterogeneity). If high levels of heterogeneity among the trials exist $\left(\mathrm{I}^{2} \geq 50 \%\right.$ or $\left.\mathrm{p}<0.1\right)$ the study design and characteristics will specifically be analysed. 
We will attempt to explain the source of heterogeneity by subgroups (study setting, study design, study country, sex distribution, reducer definitions, health status at the beginning of the follow-up period) and meta-regression analyses (baseline date, participants age, time between baseline and second CPD assessment, mean age at the end of the follow-up, duration of the follow-up period) or sensitivity analyses (risk of bias). The statistical software Comprehensive Meta-Analysis V.3.0 will be used for the meta-analysis calculations.

A systematic narrative synthesis will be provided with information presented in the text and tables to summarise and explain the characteristics and findings of the included studies. The narrative synthesis will explore the relationship and findings both within and between the included studies.

\section{Risk of bias in meta-analysis}

Publication bias assessment will be based on funnel plots. In case of asymmetry, Duval and Tweedie's Trim and Fill method will be used. ${ }^{29}$

\section{Confidence in cumulative evidence}

We will use the Grading of Recommendations, Assessment, Development and Evaluation guidelines to assess the quality of evidence for our research questions. ${ }^{30}$ This assessment of quality of evidence considers criteria subject to decrease confidence (risk of bias, imprecision, inconsistency, publication bias and heterogeneity across studies) or to increase confidence (strong association, dose-response gradient, opposing residual plausible confounding and bias). Strength of evidence will be judged as 'high' (further research is unlikely to change our conclusion), 'moderate' (further research is likely to alter our conclusion) or 'low' (further studies are required to answer the research question with a high degree of confidence/increase confidence).

\section{Patient and public involvement}

No patient or public are involved.

\section{Ethics and dissemination}

We intend to publish the results of our review in a peerreviewed journal and to present our findings at national and international meetings and conferences.

Contributors H-JA is the primary reviewer (guarantor). H-JA drafted the protocol and developed the eligibility criteria. IB conceptualised the protocol rationale. SG and LAO developed the search and the risk-of-bias assessment strategies. The data extraction form was developed by $\mathrm{H}-\mathrm{JA}, \mathrm{SG}, \mathrm{LAO}$ and IB. The manuscript was drafted by $\mathrm{H}-\mathrm{JA}$ and reviewed by SG, LAO and IB.

Funding The authors have not declared a specific grant for this research from any funding agency in the public, commercial or not-for-profit sectors.

Competing interests H-JA was member of advisory boards, DSMB, or steering committees for Pfizer, Johnson \& Johnson, Bioprojet and Ethypharm, and has received sponsorship to attend scientific meetings, speaker honoraria or consultancy fees from Bioprojet, D\&A Pharma, Ethypharm, Kinnov Pharmaceuticals, Lundbeck, and Pfizer. He is also member of the American Society of Clinical Psychopharmacology's Alcohol Clinical Trials Initiative (ACTIVE), which was supported in the last three years by Alkermes, Amygdala Neurosciences, Arbor Pharmaceuticals, Indivior, Lundbeck, Mitsubishi, and Otsuka. All outside the submitted work. Laetitia Ali Oicheih and Sonia Gabriel declare no competing interest. IB declares having received honoraria for presentations from Pfizer in the last 3 years.

Patient consent for publication Not required.

Provenance and peer review Not commissioned; externally peer reviewed.

Supplemental material This content has been supplied by the author(s). It has not been vetted by BMJ Publishing Group Limited (BMJ) and may not have been peer-reviewed. Any opinions or recommendations discussed are solely those of the author(s) and are not endorsed by BMJ. BMJ disclaims all liability and responsibility arising from any reliance placed on the content. Where the content includes any translated material, BMJ does not warrant the accuracy and reliability of the translations (including but not limited to local regulations, clinical guidelines, terminology, drug names and drug dosages), and is not responsible for any error and/or omissions arising from translation and adaptation or otherwise.

Open access This is an open access article distributed in accordance with the Creative Commons Attribution Non Commercial (CC BY-NC 4.0) license, which permits others to distribute, remix, adapt, build upon this work non-commercially, and license their derivative works on different terms, provided the original work is properly cited, appropriate credit is given, any changes made indicated, and the use is non-commercial. See: http://creativecommons.org/licenses/by-nc/4.0/.

ORCID iDs

Henri-Jean Aubin http://orcid.org/0000-0002-6604-6672

Ivan Berlin http://orcid.org/0000-0002-5928-5616

\section{REFERENCES}

1 Jha P, Peto R. Global effects of smoking, of quitting, and of taxing tobacco. N Engl J Med 2014;370:60-8.

2 Pirie K, Peto R, Reeves GK, et al. The 21st century hazards of smoking and benefits of stopping: a prospective study of one million women in the UK. Lancet 2013;381:133-41.

3 National Center for Chronic Disease P, Health Promotion Office on $\mathrm{S}$, Health. Reports of the surgeon General. The health consequences of Smoking-50 years of progress: a report of the surgeon General. Atlanta (GA): Centers for Disease Control and Prevention (US), 2014.

4 Jha P, Ramasundarahettige C, Landsman V, et al. 21St-Century hazards of smoking and benefits of cessation in the United States. $N$ Engl J Med 2013;368:341-50.

5 Banks E, Joshy G, Weber MF, et al. Tobacco smoking and allcause mortality in a large Australian cohort study: findings from a mature epidemic with current low smoking prevalence. BMC Med 2015;13:38.

6 Müezzinler A, Mons U, Gellert C, et al. Smoking and all-cause mortality in older adults: results from the chances Consortium. Am J Prev Med 2015;49:e53.

7 Glantz SA, Bareham DW. E-Cigarettes: use, effects on smoking, risks, and policy implications. Annu Rev Public Health 2018;39:215-35.

8 Inoue-Choi M, Liao LM, Reyes-Guzman C, et al. Association of long-term, low-intensity smoking with all-cause and cause-specific mortality in the National Institutes of Health-AARP diet and health study. JAMA Intern Med 2017;177:87-95.

9 Lubin JH, Couper D, Lutsey PL, et al. Risk of cardiovascular disease from cumulative cigarette use and the impact of smoking intensity. Epidemiology 2016;27:395-404.

$10 \mathrm{Hu}$ Y, Zong G, Liu G, et al. Smoking cessation, weight change, type 2 diabetes, and mortality. N Engl J Med 2018;379:623-32.

$11 \mathrm{He}$ Y, Jiang B, Li LS, et al. Changes in smoking behavior and subsequent mortality risk during a 35-year follow-up of a cohort in Xi'an, China. Am J Epidemiol 2014;179:1060-70.

12 Abrams DB, Glasser AM, Pearson JL, et al. Harm minimization and tobacco control: Reframing societal views of nicotine use to rapidly save lives. Annu Rev Public Health 2018;39:193-213.

13 Fadus MC, Smith TT, Squeglia LM. The rise of e-cigarettes, pod mod devices, and JUUL among youth: factors influencing use, health implications, and downstream effects. Drug Alcohol Depend 2019;201:85-93.

14 Rigotti NA. Balancing the benefits and harms of e-cigarettes: a national academies of science, engineering, and medicine report. Ann Intern Med 2018;168:666-7.

15 Hughes JR, Carpenter MJ. Does smoking reduction increase future cessation and decrease disease risk? A qualitative review. Nicotine Tob Res 2006;8:739-49. 
16 Pisinger C, Godtfredsen NS. Is there a health benefit of reduced tobacco consumption? A systematic review. Nicotine Tob Res 2007;9:631-46.

17 Godtfredsen NS, Holst C, Prescott E, et al. Smoking reduction, smoking cessation, and mortality: a 16-year follow-up of 19,732 men and women from the Copenhagen centre for prospective population studies. Am J Epidemiol 2002;156:994-1001.

18 Inoue-Choi M, Hartge P, Park Y, et al. Association between reductions of number of cigarettes smoked per day and mortality among older adults in the United States. Am J Epidemiol 2019;188:363-71.

19 Cho MH, Lee K, Park SM, et al. Effects of smoking habit change on all-cause mortality and cardiovascular diseases among patients with newly diagnosed diabetes in Korea. Sci Rep 2018;8:5316.

20 Hart C, Gruer L, Bauld L. Does smoking reduction in midlife reduce mortality risk? results of 2 long-term prospective cohort studies of men and women in Scotland. Am J Epidemiol 2013;178:770-9.

21 Gerber Y, Myers V, Goldbourt U. Smoking reduction at midlife and lifetime mortality risk in men: a prospective cohort study. $A m \mathrm{~J}$ Epidemiol 2012;175:1006-12.

22 Tverdal A, Bjartveit K. Health consequences of reduced daily cigarette consumption. Tob Control 2006;15:472-80.

23 Moher D, Shamseer L, Clarke M, et al. Preferred reporting items for systematic review and meta-analysis protocols (PRISMA-P) 2015 statement. Syst Rev 2015;4:1
24 Shamseer L, Moher D, Clarke M, et al. Preferred reporting items for systematic review and meta-analysis protocols (PRISMA-P) 2015: elaboration and explanation. BMJ 2015;350:g7647.

25 Wells GA BS, O'Connell D. The Newcastle-Ottawa scale (NOS) for assessing the quality of nonrandomised studies in meta-analyses Ottawa: the Ottawa Hospital research Institute, 2020. Available: http://www.ohri.ca/programs/clinical_epidemiology/oxford.asp [Accessed 12 Apr 2020]

26 Watkins C, Bennett I. A simple method for combining binomial counts or proportions with hazard ratios for evidence synthesis of time-to-event data. Res Synth Methods 2018;9:352-60.

27 Tierney JF, Stewart LA, Ghersi D, et al. Practical methods for incorporating summary time-to-event data into meta-analysis. Trials 2007;8:16.

28 Grant RL. Converting an odds ratio to a range of plausible relative risks for better communication of research findings. BMJ 2014;348:f7450.

29 Duval S, Tweedie R. Trim and fill: a simple funnel-plot-based method of testing and adjusting for publication bias in meta-analysis. Biometrics 2000;56:455-63.

30 Burford BJ, Rehfuess E, Schünemann $\mathrm{HJ}$, et al. Assessing evidence in public health: the added value of grade. $J$ Public Health 2012;34:631-5 